\title{
Etude comparative de la flore aux abords des cours d'eau dans les zones hypo et hyper endémiques d'ulcère de Buruli en Côte d'Ivoire
}

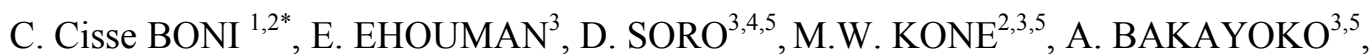 \\ F. DEMBELE ${ }^{3,5}$, K. BAUTHIRE ${ }^{3}$ et M. DOSSO ${ }^{1,2}$ \\ ${ }^{I}$ UFR des Sciences Médicales, Département de Microbiologie, Université Félix Houphouët Boigny, \\ 22 BP 582 Abidjan 22, Côte d'Ivoire. \\ ${ }^{2}$ Unité de Botanique et Médecine traditionnelle, Département environnement et santé, \\ Institut Pasteur de Côte d'Ivoire, 01 BP 490 Abidjan 01, Côte d'Ivoire. \\ ${ }^{3}$ UFR Sciences de la Nature, Université Nangui Abrogoua, 02 BP 801 Abidjan 02, Côte d'Ivoire. \\ ${ }^{4}$ UFR des Sciences biologiques, Université Péléforo Gon Coulibaly, Korhogo \\ ${ }^{5}$ Centre Suisse de Recherche Scientifique en Côte d'Ivoire. \\ *Auteur correspondant, E-mail:bonicatc@yahoo.fr
}

\section{REMERCIEMENTS}

Ce travail a été réalisé grâce à l'appui financier du Programme d'Appui Stratégique à la Recherche Scientifique (PASRES) dans le cadre de l'obtention d'une bourse.

\section{RESUME}

En Côte d'Ivoire, les foyers endémiques de l'ulcère de Buruli (UB) sont multiples. Le mode de transmission de cette infection cutanée invalidante reste encore inconnu. Les plantes aquatiques sont de plus en plus incriminées dans cette transmission. L'objectif de ce travail était de déterminer les plantes dominantes aux abords des plans d'eau des zones de forte et de faible endémicité d'UB. L'inventaire floristique a été réalisé par la méthode des relevés de surface et itinérants. La fréquence absolue, les spectres biologique et phytogéographique ont été déterminés. Les inventaires floristiques réalisés au niveau des cours d'eau dans les districts sanitaires de Tiassalé, Alépé, Divo et Yamoussoukro, ont permis de collecter plus de 47878 individus de plantes repartis en 56 familles, 166 genres et 216 espèces. Les espèces les plus fréquentes dans les sites à forte endémicité (Divo, Yamoussokro) sont Adenia cissampeloides, Pycreus flavescens, Panicum maximum, Cissus arguta, Costus afer, Ceratophyllum demersum, Amaranthus spinosus, Panicum repens et Ipomoea asarifolia. Il y a très peu de variation au niveau de spectre chorologique quelle que soit l'endémicité. Concernant le spectre biologique, les chaméphytes sont relativement plus abondants dans les sites de forte endémicité.

(C) 2017 International Formulae Group. All rights reserved.

Mots clés : Ulcère de Buruli, flore, hyper et hypo endémicité, Côte d'Ivoire.

\section{Comparative study of the flora in the fields of water courses in the hypo and hyper endemic zones of Buruli ulcer in Côte d'Ivoire}

\begin{abstract}
In Côte d'Ivoire, the endemic sites of UB are multiple. The mode of transmission of this skin infection is still unknown. The objective of this work was to determine the dominant plants in aquatic environments in
\end{abstract}


UB's high and low endemic areas. The selection of study sites was carried out with the support of the National Program for the Control of Ulcer in Buruli of Côte d'Ivoire (PNLUB). For each health district, two health centers were chosen: one with high endemicity and one with low endemicity of UB. The floristic inventory was carried out by the method of surface and walk in the bush. Absolute frequency, biological and phytogeographic spectra were determined. Floristic inventory carried out at the level of watercourses in the health districts of Tiassalé, Alépé, Divo and Yamoussoukro, collected more than 47878 individuals of plants distributed in 56 families, 166 genera and 216 species. The most frequent species in sites with high endemicity (Divo, Yamoussokro) were Adenia cissampeloides, Pycreus flavescens, Panicum maximum, Cissus arguta, Costus afer, Ceratophyllum demersum, Amaranthus spinosus, Panicum repens and Ipomoea batatas. There is very little variation in the chorological spectrum whatever the endemicity. Concerning the biological spectrum, the Chamaephytes are relatively more abundant in the sites of high endemicity.

(C) 2017 International Formulae Group. All rights reserved.

Keywords: Buruli ulcer, plant flora, hyper and hypo endemicity.

\section{INTRODUCTION}

Mycobacterium ulcerans, pathogène émergent est responsable de maladie négligée qu'est l'ulcère de Buruli (UB), une maladie de peau nécrosée, avec formation d'ulcérations importantes. Généralement, l’UB entraîne, sur les membres, d'importantes incapacités fonctionnelles invalidantes qui affectent l'homme et surtout les enfants vivants à proximité de zones humides dans les pays tropicaux (Kingsley et al., 2000 ; OMS, 2004). L'histoire et la transmission de cette mycobactérie sont encore obscures (Marsollier et al., 2007). Cependant, le rôle de l'environnement est incriminé dans cette transmission. (Williamson et al., 2012). L'UB est hyper endémique en Afrique de l'Ouest et en Afrique Centrale. Au Congo, 84911 cas ont été répertoriés de 2006 à 2010, 4651 au Ghana, 4541 cas au Bénin, 1423 au Cameroun, 982 en RDC, 11517 en Côte d'Ivoire (OMS, 2004). La transmission interhumaine n'a pas été démontrée cependant la présence d'un réservoir environnemental semble être impliqué dans cette transmission. (Tian et al., 2016). L'hypothèse de transmission environnementale suggère que M. ulcerans, qui est omniprésent dans les régions endémiques peut être directement inoculé dans le derme humain à la suite de blessures ayant lieu dans des milieux contaminés. Les études écologiques en Afrique ont trouvé M. ulcerans dans un large éventail d'échantillons environnementaux, comme le sol, les plantes, l'eau, macroinvertébrés aquatiques et vertébrés, avec des concentrations comprises entre $10^{2}$ et $10^{4}$ unités génomiques (Garchitorena et al., 2015).

La contamination de l'homme se ferait au cours des activités telles que la riziculture, la pisciculture, la pêche, les travaux champêtres, etc..... Cette transmission peut s'effectuer aussi lors de baignades et lors d'approvisionnement en eau à partir des mares ou marigots en zones endémiques (Doannio et al., 2011).

En Côte d'Ivoire, les foyers endémiques de l'UB sont multiples. Ils se répartissent sur tout le territoire. Toutefois, les régions du Centre, du Centre-Ouest, de l'Ouest, du Sud-Ouest et du Nord-Est sont particulièrement touchées. En 1998, le taux d'incidence de l'UB a atteint $22 \%$ dans certains villages. Actuellement, l'UB est la deuxième maladie due à une mycobactérie après la tuberculose et avant la lèpre (Doannio et al., 2011).

Malgré les efforts consentis, le mode de transmission de l'UB à l'homme reste encore mal connu. L'UB a été retrouvé dans l'environnement au niveau des échantillons d'eau mais pas au niveau du biofilm des plantes (Williamson et al., 2012). Il apparaît donc nécessaire d'élucider le mode de transmission de $M$. ulcerans à l'homme en vue d'améliorer les moyens de prévention de 
la maladie par la rupture de la chaîne épidémiologique. L'objectif de ce travail était de déterminer les plantes dominantes aux abords des plans d'eau des zones de forte et de faible endémicité d'UB en Côte d'Ivoire en vue d'étudier leur implication dans la transmission de M. ulcerans.

\section{MATERIEL ET METHODES}

\section{Période d'étude}

L'étude a été réalisée sur une période de 12 mois allant de septembre 2015 à septembre 2016.

\section{Sites d'étude}

La sélection des sites d'étude a été faite avec l'appui du Programme National de Lutte Contre 1'Ulcère de Buruli de Côte d'Ivoire (PNLUB). Les données relatives au nombre de cas d'UB enregistrés dans les établissements de prise en charge dans les différents districts sanitaires de la Côte d'Ivoire, pour les années 2013 et 2014, ont été fournies par le PNLUB. Pour chaque district sanitaire, deux centres de santé ont été choisis : un centre à forte endémicité et un autre à faible endémicité d'UB. Ainsi, deux zones d'étude ont été inventoriées par district sanitaire.

Les points d'eau dans chaque village ont été choisis selon deux critères :

- la fréquentation par la population

- le rapprochement du village à savoir une distance minimale de $150 \mathrm{~m}$ par rapport au village, dans un rayon de $1 \mathrm{~km}$ du centre du village (Brou et al., 2006).

Les travaux ont été réalisés sur 25 points d'eau permanent de 12 villages appartenant à huit établissements de prise en charge dans quatre districts de santé (Tableau 1, Figure 1).

Les coordonnées géographiques de chaque zone ont été enregistrées à l'aide d'un GPS de marque Garmin eTrex 30 (eTrex 30®; Garmin International, Inc., Olathe, KS, U.S.A.) avec un niveau de précision de 3 mètres. La présence ou l'absence d'activité humaine et d'animaux ont été notées.

\section{Inventaire floristique}

Les données ont été collectées dans quatre districts sanitaires: Alépé, Tiassalé, Divo et Yamoussoukro. La méthode de relevé de surface et celle de relevé itinérant ont été utilisées pour la collecte de données. La méthode de relevé de surface a consisté à délimiter une aire d'un mètre carré $(1 \mathrm{~m} \times 1$ $\mathrm{m}$ ), puis à inventorier et dénombrer les individus des espèces végétales se trouvant dans cette aire délimitée de $1 \mathrm{~m}^{2}$ (Figure 2). Les parcelles ont été positionnées à proximité des cours d'eau fréquentés par les populations. Quant à la méthode des relevés itinérants, elle a consisté à parcourir la zone des inventaires dans toutes les directions en vue de récolter des espèces non rencontrées lors des relevés de surface.

\section{Identification botanique des échantillons collectés}

Les espèces végétales ont été identifiées dans les herbiers du Centre Suisse de Recherches Scientifiques en Côte d'Ivoire et du Centre National de floristique d'Abidjan. La nomenclature utilisée dans cette étude est celle de Lebrun et Stork $(1991,1995,1997)$ et de Aké-Assi $(2001,2002)$ et de Chatelain (2011). Pour les espèces Ptéridophytes, la nomenclature utilisée est celles de TardieuBlot (1953) et White (1986).

\section{Traitement des données}

Fréquence absolue: elle représente le nombre d'individus d'une espèce végétale ligneuse inventoriée sur le site.

Spectre biologique: le spectre biologique a été établi selon la classification de Raunkiaer (1934) avec quelques adaptations proposées par Aké-Assi (2001) pour répondre aux exigences des végétations tropicales. En fonction de la taille, les espèces inventoriées ont été classées en : 
- Mégaphanérophytes (MP) : plantes de plus de $30 \mathrm{~m}$ de hauteur;

- Mésophanérophytes (mP) : plantes de 8 à 30 $\mathrm{m}$ de hauteur ;

- Microphanérophytes (mp) : plantes de 2 à 8 m de hauteur ;

- Nanophanérophytes (np) : plantes de $50 \mathrm{~cm}$ à $2 \mathrm{~m}$ de hauteur ;

- Chaméphytes (Ch) : plantes de moins de 25 $\mathrm{cm}$ de hauteur.

Spectre phytogéographique: le spectre phytogéographique est basé sur les types définis par White (1986). Les types phytogéographiques retenus sont:

- GC : Taxon commun à la région Guinéo Congolaise (zone de forêt dense humide)

- SZ: Taxon de la région Soudano Zambézienne savanes, forêts claires, steppes)

- GC- SZ : Taxon de liaison commun aux deux régions Guinéo Congolaise et Soudano Zambézienne
- GCW : Taxon endémique au bloc forestier de l'Ouest africain (Ghana, Côte d'Ivoire, Sierra Leone, Guinée, Guinée-Bissau, Gambie, Sénégal)

- i : Taxon introduit

Témoin: les zones témoins représentent les régions reconnues non endémique d'ulcère de Buruli par la PNLUB Côte d' Ivoire dans les districts sanitaires identifiés pour l'étude.

\section{Analyses statistiques}

Les données collectées ont été saisies et traitées avec le logiciel Microsoft Excel. Une liste floristique a été établie pour chaque site. Le calcul du coefficient de similitude des deux sites étudiés, à savoir le site hyperendémique et le site témoin ou hypoendémique a été fait. Sorensen (1948) a défini ce coefficient comme suit :

$\mathrm{Cs}=(2 \mathrm{C} /(\mathrm{A}+\mathrm{B})) \times 100$. Cs varie de 0 à 100 . si $\mathrm{Cs}=0$, aucune espèce en commun.

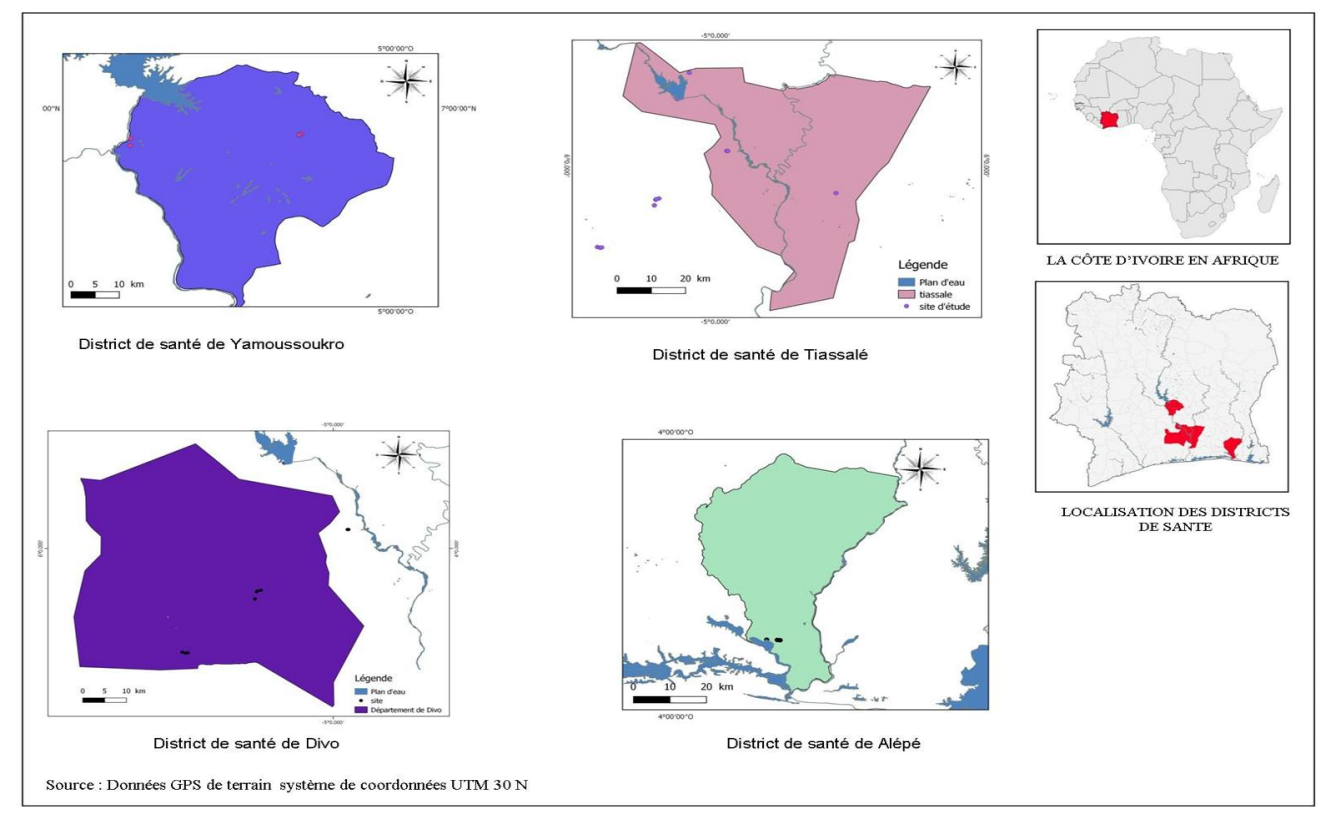

Figure 1 : Localisation des sites d'étude. 

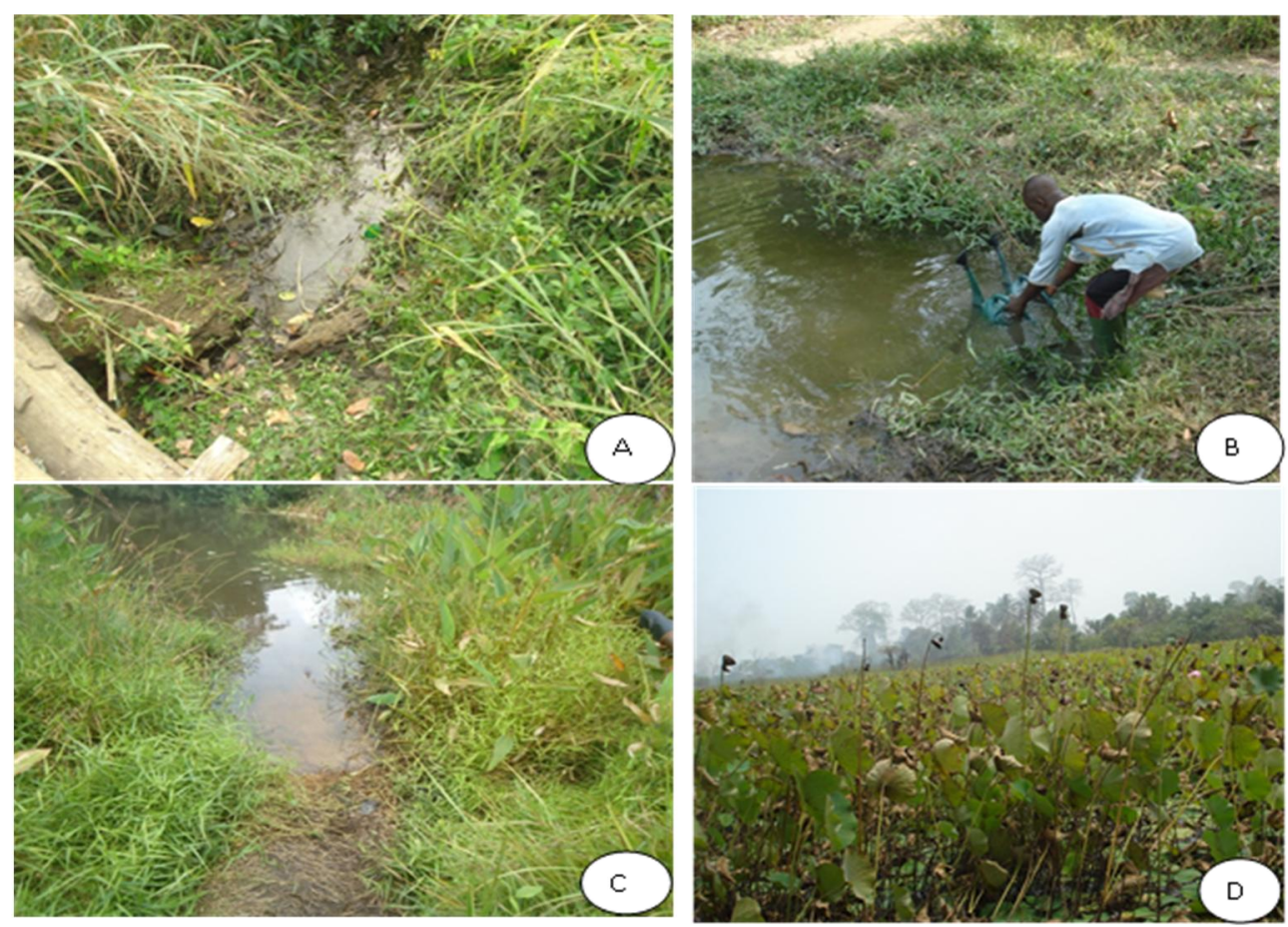

Figure 2: Différents points d'eau permanents où ont été réalisés les inventaires floristiques à Akouré (A) et à Toumbokro (B et C), peuplement de Nelumbo nucifera Gaertn (Nymphaeceae) Tombokro (D).

Tableau 1 : Districts sanitaires et sites de collecte des plantes.

\begin{tabular}{|c|c|c|c|}
\hline District sanitaire & $\begin{array}{l}\text { Etablissements de } \\
\text { prise en charge }\end{array}$ & Villages & $\begin{array}{c}\text { Nombre de quadrats } \\
\left(1 \mathrm{~m}^{2}\right)\end{array}$ \\
\hline \multirow{5}{*}{ Alépé } & \multirow{5}{*}{ Akouré } & \multirow{4}{*}{ Akouré } & 12 \\
\hline & & & 18 \\
\hline & & & 10 \\
\hline & & & 18 \\
\hline & & Ogodoumé & 10 \\
\hline \multirow{5}{*}{ Tiassalé } & \multirow[t]{2}{*}{ Sokrogbo } & \multirow{2}{*}{ Sokrogbo } & 10 \\
\hline & & & 10 \\
\hline & Bodo & Bodo & 10 \\
\hline & \multirow{2}{*}{ Taabo } & Taabo cité (Oflébo) & 41 \\
\hline & & Taabo village & 71 \\
\hline \multirow{5}{*}{ Divo } & \multirow[t]{3}{*}{ Tabléguikou } & \multirow{2}{*}{ Tabléguikou } & 20 \\
\hline & & & 24 \\
\hline & & Guilehiri & 10 \\
\hline & \multirow{2}{*}{ Ogoudou } & Ahouati & 32 \\
\hline & & Ogoudou & 13 \\
\hline \multirow{4}{*}{ Yamoussokro } & Toumbokro & & 18 \\
\hline & & Toumbokro & 90 \\
\hline & NZéré & & 30 \\
\hline & & NZéré & 40 \\
\hline Total & & 12 & 487 \\
\hline
\end{tabular}




\section{RESULTATS}

Richesse spécifique des différentes zones endémiques et non endémiques

Les inventaires floristiques réalisés au niveau des cours d'eau dans les districts sanitaires de Tiassalé, Alépé, Divo et Yamoussoukro, ont permis de collecter plus de 47878 individus de plantes repartis en 56 familles, 166 genres et 216 espèces (Tableau 2). Les familles les plus représentées sont celles des Poaceae, des Fabaceae, des Asteraceae et des Commelinaceae.

Les genres les plus riches en espèces sont : Ipomoea (8), Cyperus (7) Ludwigia (5), Commelina (4), Panicum (4). Acrocras, Adenia, Maranthus, Cola, Combretum, Croton, Cyclosorus, Dioscorea, Echinochloa, Euphorbia, Indigofera, Mimosa, Paspalum, Pennisetum, Phyllantus, Pycreus, Spilanthes et Thalia qui sont chacune représentées par au moins deux espèces. Quant aux autres genres, ils sont mono-spécifiques (Tableau 3).

Concernant le statut des sites, certaines espèces ne sont présentes que dans l'un ou l'autre de ces sites. En outre, les sites à forte endémicité à l'UB ont une richesse spécifique plus importante que ceux de faible endémicité.

\section{Espèces à forte représentation communes à} l'ensemble des sites étudiés

Pour l'ensemble des sites, il existe des espèces qui ne sont présentes que dans l'un ou dans l'autre des sites. Ainsi Ipomoea asarifolia, Nelumbo nucifera, Cyperus fertilis, Telosma africana, Ludwigia hyssopifolia sont les plus fréquentes et uniquement présentes auprès des cours d'eau dans les sites de forte endémicité. En outre Cynodon dactylon, Persicaria lanigera, Cleome rutidosperma, Culcasia saxatilis, Pycreus flavescens, Physalis angulata, Ludwigia octovalvi et Phaulopsis ciliata sont les plus fréquentes et sont uniquement présentes dans les sites hypoendémiques (Tableau 5).
Diversité floristique en fonction de la zone endémicité et de non endémicité

Dans le District sanitaire d'Alépé où l'UB est considéré comme absent, les espèces végétales les plus fréquentes sont: Synedrella nodiflora, Kyllinga erecta, Costus afer, Nymphaea lotus, Acroceras gabunens, Ipomoea batatas et Christella detata.

Les sites dans lesquels ont été enregistrés le moins de cas d'ulcère de Buruli (faible endémicité) selon PNLUB sont à dans le District sanitaire de Tiassalé, les villages de Bodo et de Taabo village, dans le District sanitaire de Divo, le village de Tabléguikou et dans celui de Yamoussoukro le village de N'zéré. Dans ces sites, quel que soit le district sanitaire considéré, les espèces végétales les plus fréquentes sont: Commelina erecta, Stylosanthes erecta, Amaranthus spinosus, Adenia cissampeloides, Sabicea discolor, Pycreus flavescens, Cynometra megalophylla, Panicum maximum et Persicaria madagascariensis.

Les sites à forte endémicité où ont été enregistrés le plus de cas d'UB sont les villages de Sokrogbo à Tiassalé, de Taabo Cité à Tiassalé, d'Ahouati à Divo, d'Ogoudou à Divo et de Toumbokro à Yamoussoukro.

Les espèces les plus fréquentes dans ces sites sont Adenia cissampeloides, Pycreus flavescens, Panicum maximum, Cissus arguta, Costus afer, Ceratophyllum demersum, Amaranthus spinosus, Panicum repens et Ipomoea batatas (Tableau 4).

\section{Spectre chorologique}

Dans les sites hypo-endémiques et hyper-endémiques, les chorologies les plus importantes sont les Guinéo-CongolaiseSoudano-Zambézienne (71) et (72) et les Guinéo-Congolaise (57) et (41).

\section{Spectre des types biologiques}

S'agissant des types biologiques, un total de 28 a été identifié. Quel que soit le site 
choisi, les naphanérophytes, les thérophytes, les lianes microphanérophytes et les chaméphytes sont les plus abondantes. Les valeurs sont de $22,95 \%$ et $22,14 \%$ pour les nanophytes ; $18,03 \%$ et $14,29 \%$ pour les thérophytes et $13,93 \%$ et $14,29 \%$ pour les lianes microphanérophytes, respectivement dans les sites de forte endémicité et les sites de faible endémicité. En plus de cette distribution, les chaméphytes sont relativement plus abondantes dans les sites de forte endémicité $(10,66 \%)$ contre $(7,86 \%)$ dans les sites de faible endémicité. Certaines des formes biologiques ne sont présentes que dans l'un ou dans l'autre des sites. Ainsi, hémicryptophyte (hydrophytes) (H Hyd), hydrophytes (Hyd), mégaphanérophytes (MP), nanophanérophytes (hémicriptophytes pyrophytiques) (np (Hpy) et stolonifère (Sto) ne sont présents bien qu'en faible proportion que dans les zones hypo-endémiques, à la différence des hydrophytes (Hyd), thérophytes (hydrophytes) (Th Hyd), géophytes rhizomateux hydrophytes (Gr Hyd), hémicryptophytes épiphytes ( $\mathrm{H} \quad \mathrm{Ep})$, et thérophytes (hémicryptophytes) Th $(\mathrm{H})$ qui ne sont présents que dans les sites hyperendémiques.

Tableau 2 : Répartition des espèces végétales selon le district sanitaire.

\begin{tabular}{lccccc}
\hline Districts sanitaires & $\begin{array}{c}\text { Nombre de } \\
\text { placettes }\end{array}$ & $\begin{array}{c}\text { Nombre } \\
\text { d'individus }\end{array}$ & $\begin{array}{c}\text { Nombre } \\
\text { de familles }\end{array}$ & $\begin{array}{c}\text { Nombre de } \\
\text { genres }\end{array}$ & $\begin{array}{c}\text { Nombre d'espèces } \\
\text { végétales }\end{array}$ \\
\hline Alépé & 68 & 2876 & 18 & 32 & 39 \\
Tiassalé & 142 & 27968 & 25 & 44 & 53 \\
Divo & 99 & 6814 & 39 & 87 & 107 \\
Yamoussokro & 178 & 10220 & 39 & 104 & 116 \\
\hline
\end{tabular}

Tableau 3: Liste des espèces inventoriées prédominantes dans les sites de fortes et de faibles endémicités.

\begin{tabular}{lccc}
\hline Espèces végétales & Forte endémicité & Faible endémicité & Témoin \\
\hline Acroceras zizanioides & 79 & 43 & 1 \\
Pueraria phaseoloides & 51 & 32 & 1 \\
Panicum laxum & 51 & 29 & 16 \\
Struchium sparganophorum & 23 & 32 & 0 \\
Alternanthera sessilis & 23 & 45 & 0 \\
Panicum maximum & 23 & 13 & 0 \\
Persicaria senegalensis & 20 & 23 & 0 \\
Lepistemon owariense & 18 & 15 & 0 \\
Ludwigia senegalensis & 16 & 9 &
\end{tabular}


Eleusine indica

Persicaria acuminata

Passiflora foetida

Ludwigia erecta

Phaseolus lunatus

Synedrela nodiflora

Phaulopsis falcisepala

Psophocarpus palustris

Sphenoclea zeylanica

Ipomoea triloba

Kyllinga erecta

Merremia umbellata

Pennisetum purpureum

Costus afer

Crinum jagus

Euphorbia hirta

Marantochloa leucantha

Thalia geniculata

Commelina diffusa

Aeschynomene afraspera

Alchornea cordifolia
15

14

10

9

7

7

7

7

6

6

6

6

5

4

4

4

4

3

3

3

3
16

7

1

0

0

0

14

0

0

0

15

0

0

0

5

0

0

0

0

29

0

0

Tableau 4: Liste des espèces inventoriées prédominantes dans les sites à forte endémicité.

\begin{tabular}{lccc}
\hline Espèces végétales & Forte endémicité & Faible endémicité & Témoin \\
\hline Acroceras zizanioides & 79 & 43 & 1 \\
Pueraria phaseoloides & 51 & 32 & 8 \\
Panicum laxum & 51 & 29 & 1 \\
Imperata cylindrica & 44 & 1 & 0 \\
Commelina erecta & 30 & 0 & 17 \\
Chromolaena odorata & 30 & 4 & 0
\end{tabular}


C.C. BONI et al. / Int. J. Biol. Chem. Sci. 11(3): 1254-1270, 2017

Centrosema pubescens

Ipomoea asarifolia

Persicaria senegalensis

Lepistemon owariense

Nelumbo nucifera

Cyperus fertilis

Ludwigia senegalensis

Telosma africana

Ageratum conyzoides

Echinochloa colona

Oldenlandia macrophylla

Ludwigia hyssopifolia

Ludwigia leptocarpa

Phyllanthus amarus

Cyclosorus oppositifolius

Cyperus haspan

Paspalum scrobiculatum

Phaseolus lunatus

Synedrela nodiflora

Phaulopsis falcisepala

Psophocarpus palustris

Sphenoclea zeylanica

Ipomoea triloba

Kyllinga erecta

Merremia umbellata

Pennisetum purpureum

Acroceras gabunense

Desmodium salicifolium

Costus afer

Sida acuta

Cissus arguta

Crinum jagus

Euphorbia hirta

Hydrolea palustris

Marantochloa leucantha

Pterocarpus santalinoides

Christella dentata

Aframomum alboviolaceum

Bacopa crenata

Cynometra megalophylla

Cyperus dilatatus
24

21

20

18

18

16

16

16

14

14

14

13

11

10

10

9

8

8

7

7

7

7

6

6

6

6

5

5

4

4

4

4

4

4

4

4

3

3

3

3

3
$3 \quad 0$

$0 \quad 0$

$23 \quad 0$

150

0

$0 \quad 0$

90

$0 \quad 0$

$3 \quad 7$

40

$6 \quad 0$

$0 \quad 0$

50

216

30

02

$1 \quad 0$

$7 \quad 0$

$6 \quad 14$

$0 \quad 0$

$0 \quad 0$

$0 \quad 0$

$0 \quad 15$

$0 \quad 0$

$0 \quad 0$

$2 \quad 0$

$0 \quad 14$

0

35

02

$\begin{array}{ll}0 & 2 \\ 0 & 0\end{array}$

30

30

0

$2 \quad 0$

$0 \quad 0$

12

0

0

$0 \quad 0$

0


Mimosa pudica

Oldenlandia corymbosa

Oplismenus hirtellus

Rottboellia exaltata

Spondias mombin

Thunbergia alata

Cardiospermum grandifolia

Crotalaria retusa

Leucaena glauca

Pennisetum polystachyon

Pycreus macrostachyos

Stylosanthes erecta

Typha domingensis

Nymphaea lotus

Albizia zygia

Asystasia gangetica

Brachiaria lata

Cassia hirsuta

Cassia mimosoides

Ceratophyllum demersum

Cola cordifolia

Cyperus difformis

Cyperus mariscus

Desmodium triflorum

Desmodium velutinum

Erigeron floribundus

Eupatorium africanum

Euphorbia heterophylla

Flueggea virosa

Gongronema latifolium

Ipomoea involucrata

Laportea aestuans

Lemna minor

Mucuna pruriens

Nephrolepis biserrata

Pancovia turbinata

Phoenix reclinata

Secamone afzelii

Sorghum arundinaceum

Spilanthes filicaulis

Tithonia diversifolia
0

0

0

0

0

10

0

0

$0 \quad 0$

0

0

0

$0 \quad 0$

0

$0 \quad 0$

0

0

$0 \quad 0$

$0 \quad 0$

0

0

$0 \quad 0$

$\begin{array}{ll}0 & 0\end{array}$

$0 \quad 0$

0

0

$\begin{array}{ll}0 & 0 \\ 0 & 0\end{array}$

$\begin{array}{ll}0 & 0 \\ 0 & 0\end{array}$

$0 \quad 0$

$0 \quad 0$

$\begin{array}{ll}0 & 0 \\ 0 & 0\end{array}$

$\begin{array}{ll}0 & 0 \\ 0 & 0\end{array}$

$0 \quad 0$

$\begin{array}{ll}0 & 0\end{array}$

$\begin{array}{ll}0 & 0 \\ 0 & 0\end{array}$

$\begin{array}{ll}0 & 0 \\ 0 & 0\end{array}$

$\begin{array}{ll}0 & 0 \\ 0 & 0\end{array}$

0

$0 \quad 0$

0


Tableau 5: Liste des espèces inventoriées prédominantes dans les sites à faible endémicité.

\begin{tabular}{|c|c|c|c|}
\hline Espèces végétales & Forte endémicité & Faible endémicité & Témoin \\
\hline Cynodon dactylon & 0 & 31 & 0 \\
\hline Persicaria lanigera & 0 & 31 & 0 \\
\hline Commelina diffusa & 3 & 25 & 29 \\
\hline Cleome rutidosperma & 0 & 23 & 0 \\
\hline Culcasia saxatilis & 0 & 23 & 0 \\
\hline Persicaria acuminata & 14 & 22 & 1 \\
\hline Rhynchospora corymbosa & 2 & 21 & 0 \\
\hline Pycreus flavescens & 0 & 20 & 0 \\
\hline Physalis angulata & 0 & 16 & 0 \\
\hline Passiflora foetida & 10 & 15 & 0 \\
\hline Ludwigia octovalvis & 0 & 14 & 0 \\
\hline Aeschynomene afraspera & 3 & 13 & 0 \\
\hline Fleurya aestuans & 1 & 13 & 0 \\
\hline Ludwigia erecta & 9 & 12 & 0 \\
\hline Alchornea cordifolia & 3 & 11 & 0 \\
\hline Phaulopsis ciliata & 0 & 11 & 0 \\
\hline Fuirena umbellata & 2 & 9 & 0 \\
\hline Stachytarpheta indica & 0 & 9 & 0 \\
\hline Dioscorea minutiflora & 0 & 8 & 0 \\
\hline Commelina capitata & 1 & 7 & 0 \\
\hline Melanthera scandens & 0 & 7 & 6 \\
\hline Pergularia daemia & 0 & 7 & 0 \\
\hline Combretum racemosum & 2 & 6 & 0 \\
\hline Panicum repens & 1 & 6 & 2 \\
\hline Raphia sudanica & 0 & 6 & 0 \\
\hline Paullinia pinnata & 2 & 5 & 0 \\
\hline Adenia lobata & 1 & 5 & 0 \\
\hline Cyperus articulatus & 0 & 5 & 3 \\
\hline Commelina benghalensis & 0 & 5 & 0 \\
\hline Ficus asperifolia & 0 & 5 & 0 \\
\hline Glinus oppositifolius & 0 & 5 & 0 \\
\hline kyllinga erecta & 2 & 4 & 4 \\
\hline Scleria depressa & 2 & 4 & 0 \\
\hline Coccinia barteri & 0 & 4 & 0 \\
\hline Colocasia esculenta & 0 & 4 & 0 \\
\hline Justicia flava & 0 & 4 & 0 \\
\hline Mollugo nudicaulis & 0 & 4 & 0 \\
\hline Ocimum gratissimum & 0 & 4 & 0 \\
\hline
\end{tabular}


C.C. BONI et al. / Int. J. Biol. Chem. Sci. 11(3): 1254-1270, 2017

Oldenlandia affinis

1

Acmella caulirhiza

Ceiba pentandra

Cyperus distans

Dalbergia afzeliana

Psychotria calva

Mallotus oppositifolius

Mikania chevalieri

Vernonia cinerea

Abrus canescens

Combretum paniculatum

Cucumis melo

Floscopa africana

Hibiscus tiliaceus

Olyra latifolia

Panicum brevifolium

Paspalum conjugatum

Phyllanthus muellerianus

Rhinacanthus virens

Ricinus communis

Setaria longiseta

Alstonia boonei

Amaranthus spinosus

Amaranthus viridis

Ampelocissus gracilipes

Anthonotha macrophylla

Astraea lobata

Cassia occidentalis

Chenopodium caudatum

Cleistopholis patens

Coix lacryma-jobi

Cola caricaefolia

Croton hirtus

Dioscorea burkilliana

Dracaena mannii

Echinochloa pyramidalis

Elaeis guineensis

Fimbristylis dichotoma

Harungana madagascariensis

Indigofera hirsuta

Indigofera podocarpa
0

0

0

0

0

1

1

0

0

0

0

0

0

0

0

0

0

0

0

0

0

0

0

0

0

0

0

0

0

0

0

0

0

0

0

0

0

0

0

0
0

0

0

0

0

0

0

0

2

0

0

0

0

0

0

0

0

0

0

0

0

0

0

0

0

0

0

0

0

0

0

0

0

0

0

0

0

0

0

0

0 
Ipomoea blepharophylla

Ipomoea lanceolata

0

Ipomoea mauritiana

0

Lecaniodiscus cupanioodes

0

Lycopersicum macrocarpum

0

Mitracarpus scaber

0

Momordica charantia

0

Nauclea latifolia

Oxalis corniculata

Pandanus candellabrum

Parquetina nigrescens

Portulaca oleaceae

Pseudospondias macrocarpa

Pterygota macrocarpa

Sabicea discolor

Sida carpunifolia

Talinum triangulare

Tristemma hirtum

Triumfetta rhomboidea

Vigna racemosa

Voacanga africana

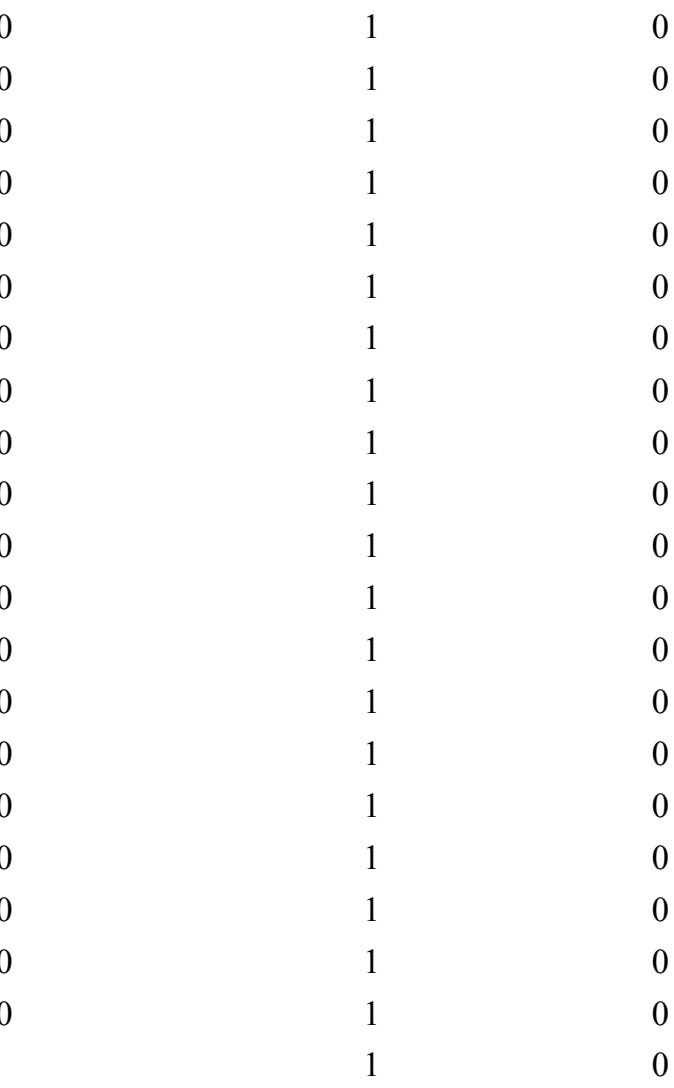

0$$
0
$$

0

0$$
0
$$$$
0
$$$$
0
$$$$
0
$$$$
0
$$$$
0
$$$$
0
$$$$
0
$$$$
0
$$$$
0
$$$$
0
$$$$
0
$$

\section{DISCUSSION}

L'UB est la troisième mycobactériose humaine après la tuberculose et la lèpre. (Portaels et al., 2008). Malgré l'amélioration de la compréhension de l'écologie des maladies infectieuses, celle de l'UB maladie ré-emergente a encore des zones d'ombre. Le pathogène $M$. ulcerans se transmettrait par effraction cutanée mais on ignore encore si la transmission directe à travers une peau humaine intacte est possible (Brou et al., 2006). Des études épidémiologiques suggèrent que les zones marécageuses, et plus spécifiquement, les milieux aquatiques, sont les principaux écosystèmes habités par $M$. ulcerans (Marsollier et al., 2007). De plus, les conditions environnementales et ses modifications, en particulier celle de la flore semble jouer un rôle important dans l'écologie de M. ulcerans (Brou et al., 2006). Les variations saisonnières du climat peuvent influencer l'écosystème de l'eau et du sol, ayant ainsi un impact sur la dynamique écologique du pathogène (Garchitorena et al., 2015). Cette bactérie a été recherchée dans plusieurs échantillons environnementaux, à savoir le sol, les détritus, le filtats des eaux de sources, les animaux aquatiques etc. (Merrittet al., 2010). Il a été montré par PCR que les végétaux, les mollusques et des poissons pouvaient être contaminés transitoirement par M. ulcerans (Marsollier et al., 2003). De plus, le biofilm de $M$. ulcerans pourrait jouer un rôle dans la transmission de l'ulcère de Buruli et dans la persistance de cette bactérie dans l'environnement. (Marsollier et al., 2007). Au niveau des animaux aquatiques certains hémiptères comme Micronecta et 
Diplonychus sont considérés comme de potentiels vecteurs de la transmission en Côte d'Ivoire (Doannio et al., 2011). En outre, des variations de positivité des échantillons d'eau et d'invertébrés aquatiques à $M$. ulcerans ont été observées en fonction de la saison (Garchitorena et al., 2014). Les travaux de Garchitonera et al. (2015), au Cameroun, ont montré que la transmission environnementale par l'inoculation non spécifique de $M$. ulcerans contaminant l'environnement peut expliquer la dynamique temporelle observée dans l'incidence de l'Ulcère de Buruli, tandis que la transmission par les punaises d'eau semble être négligeable. L'influence des variations physico-chimique de l'eau sur M.ulcerans varie en fonction des études (Pileggi et al., 2017). Cependant, aucun travail n'a fait l'inventaire de la flore où $M$. ulcerans a été isolé. Cette étude a permis de mettre en évidence la grande diversité floristique des sites endémiques à UB en Côte d'Ivoire. Cependant, très peu ou pas de travaux ne parlent de la composition floristique de ces zones. Les familles suivantes: Poaceae, Fabaceae, Commelinaceae et Melastomataceae qui représentent $47,1 \%$ dans les travaux de Noupa dans des clairières au Cameroun ont été aussi trouvées en majorité dans notre étude aussi bien en zone hyper que hypo- endémique. Cette étude constitue donc la première base des inventaires floristiques en zone d'UB en Côte d'Ivoire. Il est observé, dans ce travail, une différence dans la composition de la flore au niveau des zones hyper- endémiques et des zones hypoendémiques ayant les mêmes caractéristiques écologiques, à savoir, présence de marécages, lacs ou cours d'eau circulant lentement. Ainsi Commelina erecta, Ipomoeae asarifolia, Nelumbo nucifera, Cyperus fertilis, Telosma africana et Ludwigia hyssopifolia qui sont essentiellement retrouvées dans les zones hyper-endémiques présentent les mêmes conditions biologiques de développement que les espèces uniquement observées en zones hypo-endémique à savoir Cynodon dactylon, Persicaria lanigera, Cleome rutidosperma, Culcasia saxatilis, Pycreus flavescens, Physalis angulata, Ludwigia octovalvi et Phaulopsis ciliata. La spécificité des espèces retrouvées en zone non endémiques est leur développement pour la plupart essentiellement en touffes sur les chemins avec la formation de tapis denses sur les sommets secs. Et ceci se vérifie lors de cette étude avec Cynodon dactylon qui est une mauvaise herbe très importante des cultures non irriguées, retrouvée uniquement dans ces zones.

$\mathrm{Au}$ niveau des sites à forte endémicité, les espèces végétales, notamment Panicum maximum, Acroceras zizanioides, Pueraria phaseoloides et Imperata cylindrica présentent un aspect coupant. Elles se rencontrent le long des chemins empruntés par les populations et aux abords des point d'eau qui sont couramment visités pour diverses raisons (eaux de boisson, de lessives, de vaisselles, de toilettes). Cette particularité pourrait valider l'hypothèse émise par plusieurs chercheurs quant à la possibilité d'une contamination de l'homme par effraction cutanée. (Doannio et al., 2011; Marsollier et al., 2007). Dans cette étude, certaines espèces ne sont présentes que dans l'un ou l'autre de ces sites. En outre, les sites à forte endémicité à l'UB ont une richesse spécifique plus importante que ceux de faible endémicité. Cependant les parcelles de terre dans les deux zones ont été utilisées soit pour l'agriculture, soit pour la pêche ou un lieu où s'alimentent et s'abreuvent les animaux. Des études épidémiologiques montrent que la plupart des cas de l'UB surviennent à proximité des marécages ou dans des zones où le réseau hydrographique naturel ont été 
modifié pour les besoins de l'agriculture et de l'aquaculture (Marsollier et al., 2003). Cette pression des facteurs physiques et humains sur les sols engendre une dégradation des terres et un fort transport des charges solides vers le lit des fleuves, une pollution des eaux et une apparition des maladies dites « mystiques» selon les populations dans une étude dans le bassin béninois ( Amoussou et al., 2016) .

\section{Conclusion}

Les résultats de cette étude princeps mettent en évidence la variabilité et la densité floristique des zones hypo-endémiques et hyper-endémiques à UB. Il est à noter aussi des espèces spécifiques aux deux zones ayant pourtant les mêmes conditions écologiques de développement. La mise en relation avec une possible contamination de ces espèces spécifiques par $M$. ulcerans doit être envisagée dans une autre étude afin de pouvoir faire un lien entre cette spécificité d'espèces et la présence de M. ulcerans.

\section{CONFLITS D'INTERETS}

Les auteurs déclarent qu'il n'y a aucun conflit d'intérêt.

\section{CONTRIBUTIONS DES AUTEURS}

$\mathrm{CCB}, \mathrm{MWK}$ et $\mathrm{MD}$ ont rédigé le protocole expérimental ; $\mathrm{CCB}, \mathrm{EE}, \mathrm{DS}, \mathrm{AB}$, $\mathrm{FD}$ et $\mathrm{KB}$ ont collecté les données; $\mathrm{CCB}$, MWK, EE, DS et FD ont analysé les données; la rédaction de l'article a été réalisée par CCB, MWK, EE et DS.

\section{REFERENCES}

Aké-Assi L. 2001. Flore de la Côte-d'Ivoire : Catalogue systématique, biogéographique et écologie. Tome I. Mémoires de Botanique systématique, Boissiera 58. Conservatoire et Jardin botaniques de la Ville de Genève, 396 p.
Aké-Assi L. 2002. Flore de la Côte-d'Ivoire : Catalogue systématique, biogéographique et écologie. Tome II. Mémoires de Botanique systématique, Boissiera 58. Conservatoire et Jardin botaniques de la Ville de Genève, $401 \mathrm{p}$.

Amoussou E, Totin Vodounon S, Hougni H, A, Vissin EW, Houndenou C, Mahe G, Boko M. 2016. Changements environnementaux et vulnérabilité des écosystèmes dans le bassin-versant béninois du fleuve Niger. Int. J. Biol. Chem. Sci., 10(5): 2183-2201, DOI: http://dx.doi.org/10.4314/ijbcs.v10i5.20.

Brou T, Broutin H, Dosso M, Guegan JF. 2006. Impact des modifications d'habitats (déforestation, aménagements hydro-agricoles, extension des cultures) sur la santé des populaitions rurales : cas de l'ulcère de Buruli en Côte d'Ivoire. Climate Variability and Change Hydrological Impacts (Proceedings of the Fifth FRIEND World Conference held at Havana, Cuba, IAHS Publ. 308, 2006.

Chatelain C, Aké assi L, Rodolphe S, Laurent G. 2011. Cartes de distribution des plantes de Côte d'Ivoire, Mémoires de botanique systématique, Boissiera 64, Conservatoire et Jardin botaniques de la ville de Genève, 1-327.

Doannio JMC, Konan KL, Dosso FN, Koné AB, Konan YL. 2011. Micronecta $s p$ (Corixidae) et Diplonychus sp (Belostomatidae), deux hémiptères aquatiques hôtes et/ou vecteurs potentiels de Mycobacterium ulcerans agent pathogène de l'ulcère de Buruli en Côte d'Ivoire. Med Trop., 71: 53e-57e.
Garchitorena
A, Roche
B, Kamgang
R, Ossomba
J, Babonneau J, Landier
J, Fontanet
A, Flahault A, Eyangoh
$\mathrm{S}$, Guégan
JF, Marsollier L.
2014. 
Mycobacterium ulcerans ecological dynamics and its association with freshwater ecosystems and aquatic communities: results from a 12-month environmental survey in Cameroon. PLoS Negl. Trop. Dis., 15(5): e2879. DOI: 10.1371/journal.pntd.0002879.

Garchitorena A, Guegan JF, Leger L, Eyangoh S, Marsollier L, Roche B. 2015. Mycobacterium ulcerans dynamics in aquatic ecosystems are driven by a complex interplay of abiotic and biotic factors. eLife; 4: e07616. DOI: 10.7554/eLife.07616.

Kingsley A, Robert S, Mario R. 2000. Ulcère de Buruli : infection à Mycobacterium ulcerans. WHO/CDS/CPE/GBUI/2000.

Lebrun J-P, Stork AL. 1991. Enumération des Plantes à Fleurs d'Afrique Tropical : Généralités et Monocotylédones: Anonaceae à Pandaceae (vol 1). Edition des Conservatoires et Jardin Botaniques : Genève, Suisse ; 249.

Lebrun J-P, Stork AL. 1995. Enumération des Plantes à Fleurs d'Afrique Tropical : Généralités et Monocotylédones : Limnocharitaceae à Poaceae (vol 3). Edition des Conservatoires et Jardin Botaniques : Genève, Suisse ; 341p.

Lebrun J-P, Stork AL. 1997. Enumération des Plantes à Fleurs d'Afrique Tropical : Gamopétales : Clethraceae à Lamiaceae (vol 4). Edition des Conservatoires et Jardin Botaniques : Genève, Suisse ; $249 p$.

Marsollier L, Aubry J, Saint-André, Robert R, Legras P, Manceau L, Bourdon S, Audrain C, Carbonnelle B. 2003. Écologie et mode de transmission de Mycobacterium ulcerans Ecology and transmission of Mycobacterium ulcerans. Pathol Biol., 51(8-9): 490495.

$$
\begin{aligned}
& \text { Marsollier L; Brodin } \\
& \text { P, Jackson }
\end{aligned}
$$

Merritt RW, Walker ED, Small PL, Wallace JR, Johnson PD, Benbow ME, Boakye DA 2010. Ecology and transmission of Buruli ulcer disease: a systematic review. PLoS Negl. Trop. Dis., 14(12):e911. DOI: 10.1371/journal.pntd.0000911.

Morris AL, Guégan J-F, Andreou D, Marsollier L, Carolan K, Le Croller M, Sanhueza D, Gozlan RE. 2016. Deforestation-driven food-web collapse linked to emerging tropical infectious disease, Mycobacterium ulcerans. Sci. $A d v ., \quad$ 2: $\quad$ e1600387. DOI: $10.1126 /$ sciadv.1600387.

Noupa P, Nkongmeneck BA. 2008 .Evaluation de la diversité floristique de six clairières sur schiste dans le massif forestier de Boumba-Bek et Nki, Sud-Est Cameroun. Int. J. Biol. Chem. Sci., 2(4): 529-548. DOI : http://dx.doi.org/ 10.4314/ijbcs.v2i4. 39764.

OMS. 2004. Ulcère de Buruli. Infection à Mycobacterium ulcerans : atlas des cas déclarés dans le monde. $R E H, 279$ : 194199.

Pileggi SM, Jordan H, Clennon JA, Whitney E, Benbow ME, Merritt R, McIntosh M, Kimbirauskas R, Small P, Boakye D, Quaye C, Qi J, Campbell L, Gronseth J, Ampadu E, Opare W, Waller LA. 2017. Landscape and environmental influences on Mycobacterium ulcerans distribution 
among aquatic sites in Ghana. PLoS One. 24(4): e0176375. DOI: 10.1371/journal.pone.0176375.

Portaels F, Meyers WM, Ablordey A, Castro AG, Chemlal K, de Rijk P, Elsen P, Fissette K, Fraga AG, Lee R, Mahrous E, Small PL, Stragier P, Torrado E, Van Aerde A, Silva MT, Pedrosa J. First cultivation and characterization of Mycobacterium ulcerans from the environment. 2008. PLoS Negl Trop. Dis., 26(3):e178. DOI: 10.1371/ journal.pntd.0000178.

Raunkiaer C. 1934. The Life Forms of Plants and Statistical Plant Geography. Oxford, Clarendron Press: Oxford; 632 p.

Sorensen TA. 1948. A method of establishing groups of equal amplitude in plant sociology based on similarity of species content, and its application to analyses of the vegetation on Danish commons. Kongellige Danske Videnskabernes selskabs Biologiske Skrifer, 5: 1-34.

Tian RBD, Niamké S, Tissot-Dupont H, Drancourt M. 2016. Detection of Mycobacterium ulcerans DNA in the environment, Ivory Coast. PLoS ONE
11(3): e0151567. DOI:10.1371/journal. pone. 0151567.

Tardieu-Blot. 1953. Les Pteridophytes de l'Afrique intertropicale Français $n^{\circ} 28$. IFAN-DAKAR, Mémoire de l'Institut Française d'Afrique Noire, 241 p.

Tiebre M-S, Ouattara D, Kpangui KB, Kouassi DF, N'guessan KE. 2016. Diversité floristique de la région de Foungbesso en zone de transition forêt savane à l'ouest de la Côte d'Ivoire .Int. J. Biol. Chem. Sci., 10(3): 1007-1016. DOI : $\quad$ http://dx.doi.org/10.4314/ ijbcs.v10i3.8

White F. 1986. La Végétation de L'Afrique (The Vegetation of Africa). Orstom UNESCO, 384 pages.

Williamson HR, Benbow ME, Campbell LP, Johnson CR, Sopoh G, Barogui Y, Merritt RW, Small PL. 2012. Detection of Mycobacterium ulcerans in the environment predicts prevalence of Buruli ulcer in Benin. PLoS Negl Trop Dis., 6(1): e1506. DOI:10.1371/journal. pntd.0001506. 